\title{
Opioids and alpha-2-agonists for analgesia and sedation in newborn infants: protocol of a systematic review
}

Mari Kinoshita ${ }^{1,2^{*}}$ (D), Katarzyna Stempel ${ }^{1}$, Israel Junior Borges do Nascimento ${ }^{3,4}$, Dhashini Naidu Vejayaram', Elisabeth Norman ${ }^{5}$ and Matteo Bruschettini ${ }^{6}$

\begin{abstract}
Background: Hospitalized newborn infants may require analgesia and sedation either for the management of procedural pain, during or after surgery, and other painful conditions. The benefits and harms of opioids administered at different doses and routes of administration have been reported in numerous trials and systematic reviews. The use of alpha-2-agonists such as clonidine and dexmedetomidine in newborn infants is more recent, and they might be prescribed to reduce the total amount of opioids which are thought to have more side effects. Moreover, alpha-2-agonists might play an important role in the management of agitation and discomfort.

Methods: We will conduct a systematic review and meta-analysis on the use of opioids, alpha-2-agonists, or the combination of both drugs. We will include randomized controlled trials to assess benefits and harms and observational studies to assess adverse events and pharmacokinetics; preterm and term infants; studies on any opioids or alpha-2-agonists administered for any indication and by any route except spinal, intraosseous, or administration for nerve blocks and wound infusions. The use of opioids or alpha-2-agonists will be compared to no intervention; placebo with normal saline or other non-sedative, non-analgesic drug; control with oral sugar solution or non-pharmacological intervention; same drug of different dose or route; or a different drug (not limiting to opioids and alpha-2-agonists) or combinations of such drugs. The primary outcomes for this review will be allcause mortality during initial hospitalization and hypotension requiring medical therapy. We will conduct a search in the following databases: The Cochrane Central Register of Controlled Trials (CENTRAL, The Cochrane Library), MEDLINE, Embase, and CINAHL. Two review authors will independently screen records for inclusion, undertake data abstraction using a data extraction form and assess the risk of bias of all included trials using the Cochrane "Risk of bias" tool.
\end{abstract}

Discussion: This systematic review will summarize and update our knowledge about neonatal analgesia and sedation including pharmacokinetics/pharmacodynamics, and provide a platform for developing evidence-based guidelines that we can immediately apply to our clinical practice.

Systematic review registration: PROSPERO 2020 CRD42020170852

Keywords: Opioids, Alpha-2-agonists, Neonate, Sedation, Analgesia, Clonidine, Dexmedetomidine, Morphine, Fentanyl, Pharmacokinetics

\footnotetext{
* Correspondence: mari.kinoshita@med.lu.se

${ }^{1}$ Faculty of Medicine, Lund University, Lund, Sweden

${ }^{2}$ Keio University School of Medicine, Tokyo, Japan

Full list of author information is available at the end of the article
}

C C The Author(s). 2020 Open Access This article is licensed under a Creative Commons Attribution 4.0 International License, which permits use, sharing, adaptation, distribution and reproduction in any medium or format, as long as you give appropriate credit to the original author(s) and the source, provide a link to the Creative Commons licence, and indicate if changes were made. The images or other third party material in this article are included in the article's Creative Commons licence, unless indicated otherwise in a credit line to the material. If material is not included in the article's Creative Commons licence and your intended use is not permitted by statutory regulation or exceeds the permitted use, you will need to obtain permission directly from the copyright holder. To view a copy of this licence, visit http://creativecommons.org/licenses/by/4.0/ The Creative Commons Public Domain Dedication waiver (http://creativecommons.org/publicdomain/zero/1.0/) applies to the data made available in this article, unless otherwise stated in a credit line to the data. 


\section{Background}

\section{Description of the condition}

Newborn infants in the neonatal intensive care unit (NICU) are not just ill but constantly exposed to various stimuli through repeated invasive procedures, physical handling, and the extrauterine environment itself for those that are born preterm. A systematic review in 2008 looking into the number of painful procedures reported that a NICU patient received a median of 10 (interquartile range (IQR) 5-17) per day during the first 2 weeks of hospitalization; a later review in 2016 reported a similar average of 7.5 to 17.3 per day, and the 8 years difference in publication did not change the fact that the procedures were often accompanied by inconsistent and inadequate analgesia [1,2]. All these stimuli result in acute pain and stress, and also easily mount to chronic pain due to hyperalgesia during a vital period of complex brain development. The plasticity of the neonatal brain increases its vulnerability to these early adverse events, thereby leading to abnormal neurodevelopmental, behavioral, and cognitive outcomes [3-8]. Moreover, premature infants with even more immature brains are already predisposed to developing such sequelae from inadequately treated pain, while being most likely to be exposed to more pain during their longer NICU hospitalization. The unique characteristic of the neonatal population strengthens the rationale to establish a therapeutic approach for adequate analgesia and sedation.

\section{Description of the intervention}

Various interventions to reduce pain have been studied, but clinical protocols and guidelines still differ a lot. For short, mild to moderately painful procedures, the use of non-pharmacological strategies should always be considered [9]. For moderate to severe painful conditions, opioids have traditionally been used in the NICU, although with several side effects such as respiratory depression, hypotension, constipation, and also development of tachyphylaxis and abstinence. Opioids are also often used for sedation to reduce stress during mechanical ventilation.

The opioids most often used during neonatal intensive care are morphine, fentanyl, and remifentanil. The fentanyl derivates alfentanil and sufentanil are more randomly used. The opioids have varying pharmacokinetic (PK) and pharmacodynamic (PD) profiles and should optimally be administered in an individualized way according to the need, clinical state, and expected development of the hospitalization. Fentanyl and remifentanil are administered intravenously in the very sick infants, whereas morphine can be administered by both intravenous and oral routes.
Morphine has the longest duration of onset, half-life, and elimination time, followed by fentanyl and remifentanil [10-12]. Remifentanil is a short-acting opioid with ultra-rapid onset and very fast elimination profile, very suitable for rapid painful procedures such as tracheal intubations [13]. PD studies on opioids report on hypotension as the most adverse effect [10]. Several larger studies have questioned the effect and reported on a negative outcome [14-16] and study data report on the negative impact on the structure and function of the developing brain including neuronal apoptosis [17-19].

To reduce the opioid doses, additive treatment with paracetamol and alpha-2-agonists (clonidine and dexmedetomidine) has been implemented in the NICU [20, $21]$. The analgesic and sedative properties of the alpha2-agonists without respiratory and gastrointestinal side effects, along with the potential beneficial effects to the immature brain, have promoted their use to enable reduced opioid dosage or even to replace opioid administration [21-25].

Clonidine is administered intravenously or orally, and it was initially used to treat neonatal opioid abstinence [26]. Clonidine reduces fentanyl and midazolam demand by inducing deeper levels of analgesia and sedation without substantial side effects in ventilated newborn infants [23].

Dexmedetomidine is intravenously administered. There are dexmedetomidine PK data in newborns indicating longer half-lives, lower clearance, and smaller doses required for adequate effects in infants of lower age $[27,28]$. Study data on dexmedetomidine report selflimited bradycardia and hypotension which do not require discontinuation of the drug: dexmedetomidine should be considered as safe in this population [21, 2731]. In studies of dexmedetomidine used in combination with opioids for postoperative care, opioid-sparing effects are reported [21, 29].

Electroencephalogram (EEG) has been used to evaluate sedative and analgesic drug effects to the brain (newborn brain), and show significant background depression [32] and in some cases epileptiform activity and seizures [33, 34]. In some of the studies, neurodepression is related to drug-induced negative hemodynamic changes as assessed by near-infrared spectroscopy (NIRS) [35].

Aiming at more individualized pharmacologic treatment, pharmacogenetic studies are needed. Singlenucleotide polymorphisms in genes involved in pain control might predispose to exaggerated sensitivity or difference in opioid analgesic effect [12].

\section{How the intervention might work}

Opioids work by binding to mu, kappa, and delta-opioid receptors and modulating nociceptive information at both peripheral and central sites, thus promoting a 
temporary pain relief that is dose dependent and well tolerated [36]. In addition to analgesic effects, opioids demonstrate sedative effects through generalized central nervous depression though its precise mechanism remains unknown.

In contrast, dexmedetomidine and clonidine are alpha2 -agonists that have analgesic and sedative properties that work through a completely different mechanism involving the brainstem and the spinal cord. Dexmedetomidine is eight to ten times more specific for alpha-2adrenergic receptors than clonidine [37], but both drugs act by stimulating presynaptic alpha-2-adrenoceptors which consequently decreases synaptic noradrenaline concentrations. Inhibitory signals are transmitted from locus coeruleus in the brainstem through the descending reticulospinal tracts, as well as from dorsal horn neurons, and thus reducing substance P [38]. Since opioids and alpha-2-agonists are different types of analgosedatives, the combination of both drugs can be used to reduce doses of the drug with anticipated side effects.

\section{Why is it important to do this review}

Inadequate pain management in early human life leads to neurologic and neuropsychologic sequelae, while exposure to analgosedatives during the same period is associated with neuroapoptosis in animal experiments and affected neurodevelopment in human studies [18, 19]. Both aspects contribute to the impaired neurodevelopmental outcome; therefore, the current practice of analgosedatives remains to be reconsidered and tailored to reach effective control of neonatal pain with minimal harm to the most vulnerable patients. There is a call for a systematic review with the available evidence on opioids and alpha-2-agonists to better provide clinical guidance for neonatal pain management.

\section{Objectives}

The aim of this systematic review is to summarize the best available evidence regarding the use of opioids, alpha-2-agonists, or the combination of both for the optimal management of pain and distress in critically ill infants in the NICU.

\section{Methods/design}

We will conduct a systematic review using the standard methods of the Cochrane Neonatal Review Group (neonatal.cochrane.org/resourcesauthors/author-resourcesnew-reviews). The protocol for this review has been registered to the International Prospective Register of Systematic Reviews (PROSPERO; CRD42020170852) and will follow the reporting guideline by the Preferred Reporting Items for Systematic Review and MetaAnalyses for Protocols (PRISMA-P) (Additional file 1).
There is no need for an ethical approval for this study due to the inherent design of a systematic review.

\section{Types of studies}

We will include all randomized and quasi-randomized controlled trials (RCTs), observational studies if reporting pharmacokinetics data. Cross-over and clusterrandomized trials will be excluded.

\section{Types of participants}

Our population of interest are preterm and term infants of a postmenstrual age (PMA) up to 46 weeks and 0 days, irrespective of their gestational age at birth. We will exclude data on infants who are already receiving sedative or analgesic drugs, infants treated for neonatal abstinence syndrome, and infants undergoing dialysis. If a study enrolls patients both within and outside of our established target population, i.e., infants up to PMA 46 weeks and 0 days and those above PMA 46 weeks and 1 day are included together in the study, we will attempt to contact the authors of said study in order to obtain full data for each age group and include only data concerning infants that meet the inclusion criteria.

\section{Types of interventions}

We will include studies on any opioids, alpha-2-agonists (i.e., clonidine and dexmedetomidine), or the combination of both administered for any indication and by any other route than spinal (i.e., intrathecal, epidural, caudal) or intraosseous, also excluding administration for nerve blocks and wound infusions. The comparators or controls are no intervention; placebo with normal saline or other non-sedative, non-analgesic drug; control with oral sugar solution or non-pharmacological intervention (skin-to-skin contact, music exposure, non-nutritive sucking, swaddling, etc.); same drug of different dose or route; or a different drug (including drugs other than opioids and alpha-2-agonists) or combinations of such drugs.

\section{Types of outcome measures: primary outcomes, secondary outcomes}

The primary outcomes for this review are all-cause mortality during initial hospitalization, and circulatory instability, defined as hypotension requiring medical therapy (vasopressors, fluid boluses, or any changes in such drug administration).

The secondary outcomes measured are all-cause neonatal mortality (death until postnatal day 28); episodes of bradycardia defined as a fall in heart rate of more than $30 \%$ below the baseline or less than 100 beats per minute for $10 \mathrm{~s}$ or longer, and respiratory depression, that is episodes of apnea; neonatal pain and sedation during the administration of selected drugs, assessed 
with validated scales for procedural pain (the Neonatal Facial Coding System (NFCS) [39], the Neonatal Infant Pain scale (NIPS) [40], the Premature Infant Pain Profile (PIPP and PIPP-r) [41, 42], the Neonatal Pain, Agitation and Sedation scale (N-PASS) [43], and continuous pain (the CRIES (acronym of Crying, Requires oxygen, Increased vital signs, Expression, Sleepless) scale [44], the Échelle Douleur Inconfort Nouveau-né (EDIN) scale [45], the Neonatal Pain, Agitation, and Sedation scale (N-PASS) [43], COMFORT-B [46], the COMFORTneo [47], and the Astrid Lindgren and Lund Children's Hospital's Pain and Stress Assessment scale for Preterm and Sick Newborn Infants (ALPS-Neo) [48]); retinopathy of prematurity; intraventricular hemorrhage; necrotizing enterocolitis; periventricular leukomalacia; bronchopulmonary dysplasia; constipation, and finally cerebral NIRS and aEEG. We will also include data on the duration of mechanical ventilation, oxygen supplementation, hospital stay, and the time to full enteral feeding. Furthermore, we will measure the frequency of major neurodevelopmental disabilities: cerebral palsy, developmental, intellectual impairment, blindness, or sensorineural deafness requiring amplification. For these, we plan to evaluate each of the components as a separate outcome and extract data on each long-term outcome from studies that evaluated children after 18 months' chronological age, assessing data on children 18 to 24 months of age and on those 3 to 5 years of age separately. We will also include pharmacokinetic data (from observational studies): half-life, elimination time, and other reported pharmacokinetic measures.

\section{Search methods for identification of studies}

We will conduct a systematic literature review search in the following databases: The Cochrane Central Register of Controlled Trials (CENTRAL, The Cochrane Library), MEDLINE (Ovid), and Embase and CINAHL for eligible studies to be included (Additional file 2). We will search https://clinicaltrials.gov and ICTRP for ongoing trials. No language and time restrictions will be applied.

\section{Searching other resources}

Additionally, we will review the reference lists of all identified articles for any relevant articles that will not be identified in the primary search.

\section{Selection of studies}

Two review authors will independently screen the titles and abstracts to identify potentially relevant citations, retrieve the full texts of all potentially relevant articles, and assess the eligibility of the studies. We will resolve any disagreements by discussion and, if necessary, by consulting a third review author. We will provide details of studies excluded from the review along with the reasons for exclusion. We will contact the trial authors if the details of the primary trials are unclear to request further information.

\section{Data extraction and management}

Two review authors will independently undertake data abstraction using a data extraction form. We will extract the following characteristics from each included study:

- Administrative details: author(s), whether published or unpublished, year of publication, year in which study was conducted

- Details of study: study design, type, duration, and completeness of follow-up (e.g., greater than 80\%), country and location of study informed consent, and ethics approval

- Details of participants: birth weight, gestational age, and number of participants

- Details of intervention: modality of administration and dose of the drugs

- Details of outcomes, as listed in types of outcome measures

We will resolve any disagreement by discussion between the review authors.

We will describe any ongoing studies identified, detailing the primary author, methods, and outcome measures.

If queries arise or when additional data are required, we will contact the authors of the trial reports. Two review authors will use the Review Manager 5 software (ReviewManager 2014, RevMan 5.4) to enter all the data [49].

\section{Assessment of risk of bias in included studies}

Two review authors will independently assess the risk of bias (low, high, or unclear) of all included trials using the Cochrane "Risk of Bias" tool for the following domains [50]:

- Sequence generation (selection bias)

- Allocation concealment (selection bias)

- Blinding of participants and personnel (performance bias)

- Blinding of outcome assessment (detection bias)

- Incomplete outcome data (attrition bias)

- Selective reporting (reporting bias)

- Any other bias

We will resolve any disagreements by discussion or through a third review author.

For the included observational studies, we will use the "Risk Of Bias In Non-randomised Studies - of Interventions" (ROBINS-I) tool [51] to formally assess the risk of 
bias in the following domains: bias due to confounding, bias in selection of participants into the study, bias in classification of interventions, bias due to deviations from intended interventions, bias due to missing data, bias in measurement of outcomes, bias in the reported results, and the overall risk of bias. In the confounding domain, we took into account the following confounding factors: antenatal steroids, gestational age, birth weight, sex, Apgar score, antenatal exposure to opioids, indication to start opioids, and level of respiratory support at study entry.

\section{Measures of treatment effect}

In general, we will extract categorical data for each intervention group and calculate risk ratios (RRs) and absolute risk differences (RDs). We will obtain means and standard deviations (SDs) for continuous data, and perform analyses using mean differences (MDs) when studies were measured in the same way across trials. We will use the standardized mean difference (SMD) to combine trials that measured the same outcome but used different methods. Where trials reported continuous outcomes as median and IQR and the data passed the test of skewness, we will convert mean to median and estimate the $\mathrm{SD}$ as $\mathrm{IQR} / 1.35$. If we find a variety of scales across studies, subgroup analysis will be performed pooling these measurements. For each measure of effect, we will calculate the corresponding 95\% confidence intervals (CIs).

To specifically describe for the two primary outcomes, all-cause mortality during initial hospitalization will be assessed as RRs or RDs calculated from the numbers of infants who did or did not survive in each study arm. Hypotension requiring medical therapy (vasopressors, fluid boluses, or any changes in such drug administration) will also be assessed as RRs or RDs calculated from the number of infants who did or did not require such therapies in each study arm.

\section{Unit of analysis issues}

The unit of analysis will be the individual infant. For multiple painful procedures, we will consider the first procedure performed in the randomized infant.

\section{Dealing with missing data}

We will contact the original study investigators to request additional data where information about critical and important outcomes will be missing. We will investigate attrition rates (e.g., dropouts, losses to follow-up, and withdrawals). We will perform a sensitivity analysis to evaluate the overall results with and without the inclusion of studies with significant dropout rates. If a study will report outcomes only for participants completing the trial, or only for participants who followed the protocol, we will contact the authors and ask them to provide additional information to facilitate an intention-to-treat analysis. We will address the potential impact of missing data on the findings of the review in the "Discussion" section of the final manuscript.

\section{Assessment of heterogeneity}

We will assess clinical heterogeneity by comparing the distribution of important participant factors between trials and trial factors (e.g., randomization concealment, blinding of outcome assessment, loss to follow-up, treatment type, co-interventions). We will assess statistical heterogeneity by examining the $I^{2}$ statistic [50], a quantity that describes the proportion of variation in point estimates that is due to variability across studies rather than sampling error.

We will interpret the $I^{2}$ statistic as described by Higgins 2003 [52]:

- Less than $25 \%$ : no heterogeneity

- $25 \%$ to $49 \%$ : low heterogeneity

- $50 \%$ to $74 \%$ : moderate heterogeneity

- $75 \%$ or greater: high heterogeneity

In case of high heterogeneity ( $I^{2}$ equal to or greater than $75 \%$ ), we will not pool the studies in the metaanalysis. In addition, we will employ the chi $^{2}$ test of homogeneity to determine the strength of evidence that heterogeneity is genuine. We will explore clinical variation across studies by comparing the distribution of important participant factors among trials and trial factors (randomization concealment, blinding of outcome assessment, loss to follow-up, treatment type, and cointerventions). We will consider a threshold $P$ value of less than 0.1 as an indicator of whether heterogeneity (genuine variation in effect sizes) is present.

\section{Assessment of reporting biases}

We will create and examine a funnel plot to explore possible small-study biases. In interpreting funnel plots, we will examine the different possible reasons for funnel plot asymmetry as outlined in section 10.4 of the Handbook and relate this to the results of the review. If we are able to pool more than 10 trials, we will undertake formal statistical tests to investigate funnel plot asymmetry, and will follow the recommendations in section 10.4 of the Handbook $[50,53]$.

\section{Data synthesis}

We will perform statistical analyses according to the recommendations of the Cochrane Neonatal Review Group (neonatal.cochrane.org/en/index.html). We will analyze all infants randomized on an intention-to-treat basis. For any meta-analyses, we will synthesize data using RR, RD, 
number needed to treat to benefit (NNTB), number needed to treat to harm (NNTH), MD, and 95\% CI. For our two primary outcomes, we will assess whether the mean and $95 \%$ CI of overall RRs are below or above the null of 1 . We will analyze and interpret individual trials separately when we judged meta-analysis to be inappropriate.

\section{Subgroup analysis and investigation of heterogeneity}

If enough RCTs will be included, we will perform a subgroup analysis for the following:

- Gestational age

- Postnatal age

- Indication (e.g., sedation vs analgesia)

- Type of pain (e.g., procedural, postoperative, or other painful condition, sedation for mechanical ventilation)

- Type of administration (loading dose or not, bolus vs continuous infusion)

- Route of administration (enteral vs intravenous, between other routes)

- Specific conditions affecting pharmacokinetics (e.g., on extracorporeal membrane oxygenation (ECMO) or not, on hypothermia or not)

- Combination of administration (effect of target drug compared to a specific drug or combination of drugs)

\section{Sensitivity analysis}

We will conduct sensitivity analyses to explore the effect of the methodological quality of the trials, checking to ascertain if studies with a high risk of bias overestimated the effect of treatment.

\section{Summary of findings and assessment of the certainty of the evidence}

We planned to use the GRADE approach, as outlined in the GRADE Handbook to assess the quality of evidence for the following clinically relevant outcomes (gdt.guidelinedevelopment.org/central_prod/_design/client/handbook/handbook.html) [54]:

1. All-cause neonatal mortality (death until postnatal day 28)

2. All-cause mortality during initial hospitalization

3. Hypotension requiring medical therapy (vasopressors, fluid boluses, or any changes in such drug administration)

4. Episodes of bradycardia defined as a fall in heart rate of more than $30 \%$ below the baseline or less than 100 beats per minute for $10 \mathrm{~s}$ or longer

5. Respiratory depression, i.e., episodes of apnea (mean rates of apnea)
6. Pain during the administration of selected drugs

7. Sedation during the administration of selected drugs

Two review authors will independently assess the certainty of the evidence for each of the outcomes above. We will consider evidence from RCTs as high certainty but downgrade the evidence one level for serious (or two levels for very serious) limitations based upon the following: design (risk of bias), consistency across studies, directness of the evidence, precision of estimates, and presence of publication bias. We will use the GRAD Epro GDT software to create a "Summary of findings" table to report the certainty of the evidence.

The GRADE approach results in an assessment of the certainty of a body of evidence as being one of the following four grades:

- High: we are very confident that the true effect lies close to that of the estimate of the effect

- Moderate: we are moderately confident in the effect estimate; the true effect is likely to be close to the estimate of the effect, but there is a possibility that it is substantially different

- Low: our confidence in the effect estimate is limited; the true effect may be substantially different from the estimate of the effect

- Very low: we have very little confidence in the effect estimate; the true effect is likely to be substantially different from the estimate of effect

\section{Discussion}

Since the late 1990s, there has been a huge interest in the domain of pain-related issues in the newborn population. A large amount of studies with different study designs and varying quality has been conducted in different areas including microbiology, pharmacology, neurophysiology, and caring strategies. "The EU Regulation on medical products for paediatric use" came into use in 2007, stating that all drugs used should have been studied on the specific patient population to which they are administered [55], and this started an era of intensive research in neonatal pharmacology. Since then, the pharmacokinetic modeling [56] has allowed to expand the research opportunities in neonatal medicine, e.g., the enhanced possibilities for pharmacokinetic/pharmacodynamic (PK/PD) studies.

However, the situation varies in the NICU; the infants differ in regard to gestational and postnatal age, illness, diagnoses, and most of all the type of clinical care varies between hospitals and countries. The need for evidencebased national and international guidelines is of utmost importance. The optimal platform for developing safe 
recommendations and such guidelines would be initiated by a systematic review.

\section{Supplementary information}

Supplementary information accompanies this paper at https://doi.org/10. 1186/s13643-020-01436-0.

Additional file 1:. PRISMA-P checklist

Additional file 2:. Search strategy used for online databases

\section{Abbreviations}

NICU: Neonatal intensive care unit; IQR: Interquartile range;

PK: Pharmacokinetics; PD: Pharmacodynamics; EEG: Electroencephalography; NIRS: Near-infrared spectroscopy; RCT: Randomized controlled trial; PMA: Postmenstrual age; aEEG: Amplitude integrated electroencephalography; RR: Risk ratio; RD: Risk difference; SD: Standard deviation; MD: Mean difference; SMD: Standardized mean difference; $\mathrm{Cl}$ : Confidence interval; NNTB: Number needed to treat to benefit: NNTH: Number needed to treat to harm

\section{Acknowledgements}

The authors would like to thank Maria Bjorklund (Cochrane Sweden; Library and ICT services, Lund University) for designing the search strategy used in this review.

\section{Authors' contributions}

$M K, I B, D V, E N$, and $M B$ conceived and refined the idea for this review; MK, IB, $D V$, and $M B$ discussed the design of the search strategy; $M K, E N$, and $M B$ designed the protocol; and $\mathrm{MK}, \mathrm{KS}, \mathrm{IB}, \mathrm{EN}$, and $\mathrm{MB}$ participated in the drafting of the manuscript. All authors have read and approved the final manuscript.

\section{Funding}

The following team members have posts/work supported by external funding:

DV: Swedish Research Council (2014-07113)

EN: Swedish Research Council (2014-07113) and Region Skåne research grant MB: Governmental ALF research grants to Lund University and Lund University Hospital (2019-YF0035)

Open access funding provided by Lund University.

\section{Availability of data and materials}

Not applicable.

Ethics approval and consent to participate Not applicable.

\section{Consent for publication}

Not applicable.

\section{Competing interests}

The authors declare that they have no competing interests.

\section{Author details}

${ }^{1}$ Faculty of Medicine, Lund University, Lund, Sweden. ${ }^{2}$ Keio University School of Medicine, Tokyo, Japan. ${ }^{3}$ School of Medicine at Universidade Federal de Minas Gerais, Minas Gerais, Belo Horizonte, Brazil. ${ }^{4}$ Medical College of Wisconsin, Milwaukee, WI, USA. ${ }^{5}$ Lund University, Skane University Hospital, Department of Clinical Sciences Lund, Pediatrics, Lund, Sweden. ${ }^{6}$ Cochrane Sweden, Skåne University Hospital, Lund, Sweden.

Received: 11 May 2020 Accepted: 29 July 2020

Published online: 20 August 2020

\section{References}

1. Carbajal R, Rousset A Fau - Danan C, Danan C Fau - Coquery S, Coquery S Fau - Nolent P, Nolent P Fau - Ducrocq S, Ducrocq S Fau - Saizou C, et al. Epidemiology and treatment of painful procedures in neonates in intensive care units. JAMA. 2008;300(1):60-70.
2. Cruz MD, Fernandes AM, Oliveira CR. Epidemiology of painful procedures performed in neonates: a systematic review of observational studies. Eur J Pain. 2016;20(4):489-98.

3. Anand KJ. Clinical importance of pain and stress in preterm neonates. Bio Neonate. 1998;73(1):1-9.

4. Anand KJ, Scalzo FM. Can adverse neonatal experiences alter brain development and subsequent behavior? Biol Neonate. 2000;77(2):69-82.

5. Brummelte S, Grunau Re Fau - Chau V, Chau V Fau - Poskitt KJ, Poskitt Kj Fau - Brant R, Brant R Fau - Vinall J, Vinall J Fau - Gover A, et al. Procedural pain and brain development in premature newborns. Ann Neurol. 2012; 71(3):385-396

6. Ranger M, Grunau RE. Early repetitive pain in preterm infants in relation to the developing brain. Pain Manag. 2014;4(1):57-67.

7. Duerden EG, Grunau RE, Guo T, Foong J, Pearson A, Au-Young S, et al. Early procedural pain is associated with regionally-specific alterations in thalamic development in preterm neonates. J Neurosci. 2018;38(4): 878-86

8. Vinall J, Grunau RE. Impact of repeated procedural pain-related stress in infants born very preterm. Pediatr Res. 2014;75(5):584-7.

9. Bucsea O, Pillai RR. Non-pharmacological pain management in the neonatal intensive care unit: managing neonatal pain without drugs. Semin Fetal Neonatal Med. 2019;24(4):101017.

10. Thigpen JC, Odle BL, Harirforoosh S. Opioids: a review of pharmacokinetics and pharmacodynamics in neonates, infants, and children. Eur J Drug Metab Pharmacokinet. 2019:44(5):591-609.

11. Ziesenitz VC, Vaughns JD, Koch G, Mikus G, van den Anker JN. Correction to: pharmacokinetics of fentanyl and its derivatives in children: a comprehensive review. Clin Pharmacokinet. 2018;57(3):393-417.

12. Van Donge T, Mian P, Tibboel D, Van Den Anker J, Allegaert K. Drug metabolism in early infancy: opioids as an illustration. Expert Opin Drug Metab Toxicol. 2018;14(3):287-301.

13. McPherson C. Premedication for endotracheal intubation in the neonate. Neonatal Netw. 2018;37(4):238-47.

14. Simons $\mathrm{SH}$, van Dijk M, van Lingen RA, Roofthooft $\mathrm{D}$, Duivenvoorden $\mathrm{HJ}$, Jongeneel $\mathrm{N}$, et al. Routine morphine infusion in preterm newborns who received ventilatory support: a randomized controlled trial. Jama. 2003; 290(18):2419-27.

15. Hall RW, Kronsberg SS, Barton BA, Kaiser JR, Anand KJ. Morphine, hypotension, and adverse outcomes among preterm neonates: who's to blame? Secondary results from the NEOPAIN trial. Pediatrics. 2005:115(5): $1351-9$

16. Anand KJ, Hall RW, Desai N, Shephard B, Bergqvist LL, Young TE, et al. Effects of morphine analgesia in ventilated preterm neonates: primary outcomes from the NEOPAIN randomised trial. Lancet. 2004;363(9422):167382.

17. Sanders RD, Hassell J, Davidson AJ, Robertson NJ, Ma D. Impact of anaesthetics and surgery on neurodevelopment: an update. Br J Anaesth. 2013;110 Suppl 1(Suppl 1):153-72.

18. McPherson C, Haslam M, Pineda R, Rogers C, Neil JJ, Inder TE. Brain injury and development in preterm infants exposed to fentanyl. Ann Pharmacother. 2015:49(12):1291-7.

19. Zwicker JG, Miller SP, Grunau RE, Chau V, Brant R, Studholme C, et al. Smaller cerebellar growth and poorer neurodevelopmental outcomes in very preterm infants exposed to neonatal morphine. J Pediatr. 2016;172:817.e2.

20. Romantsik O, Calevo MG, Norman E, Bruschettini M. Clonidine for sedation and analgesia for neonates receiving mechanical ventilation. Cochrane Database Syst Rev. 2017;5(5):Cd012468.

21. Sellas MN, Kyllonen KC, Lepak MR, Rodriguez RJ. Dexmedetomidine for the management of postoperative pain and sedation in newborns. J Pediatr Pharmacol Ther. 2019;24(3):227-33.

22. Alam A, Suen KC, Hana Z, Sanders RD, Maze M, Ma D. Neuroprotection and neurotoxicity in the developing brain: an update on the effects of dexmedetomidine and xenon. Neurotoxicol Teratol. 2017:60:102-16.

23. Hünseler C, Balling G Fau - Röhlig C, Röhlig C Fau - Blickheuser R, Blickheuser R Fau - Trieschmann U, Trieschmann U Fau - Lieser U, Lieser U Fau - Dohna-Schwake C, et al. Continuous infusion of clonidine in ventilated newborns and infants: a randomized controlled trial. Pediatric critical care medicine: a journal of the Society of Critical Care Medicine and the World Federation of Pediatric Intensive and Critical Care Societies. 2014. 15(6):511-522 
24. Laudenbach V, Mantz J, Lagercrantz H, Desmonts JM, Evrard P, Gressens P. Effects of alpha(2)-adrenoceptor agonists on perinatal excitotoxic brain injury: comparison of clonidine and dexmedetomidine. Anesthesiology. 2002;96(1):134-41.

25. Pontén E, Viberg $H$, Gordh T, Eriksson P, Fredriksson A. Clonidine abolishes the adverse effects on apoptosis and behaviour after neonatal ketamine exposure in mice. Acta Anaesthesiol Scand. 2012;56(8):1058-65.

26. Broome L, So TY. Neonatal abstinence syndrome: the use of clonidine as a treatment option. NeoReviews. 2011;12(10):e575-84.

27. Chrysostomou C, Schulman SR, Herrera Castellanos M, Cofer BE, Mitra S, da Rocha MG, et al. A phase II/III, multicenter, safety, efficacy, and pharmacokinetic study of dexmedetomidine in preterm and term neonates. J Pediatr. 2014;164(2):276-82.e1-3.

28. Greenberg RG, Wu H, Laughon M, Capparelli E, Rowe S, Zimmerman KO, et al. Population pharmacokinetics of dexmedetomidine in infants. J Clin Pharmacol. 2017;57(9):1174-82.

29. Dersch-Mills DA, Banasch HL, Yusuf K, Howlett A. Dexmedetomidine use in a tertiary care NICU: a descriptive study. Ann Pharmacother. 2019;53(5):46470.

30. Estkowski LM, Morris JL, Sinclair EA. Characterization of dexmedetomidine dosing and safety in neonates and infants. J Pediatr Pharmacol Ther. 2015; 20(2):112-8

31. O'Mara K, Gal P, Wimmer J, Ransom JL, Carlos RQ, Dimaguila MA, et al. Dexmedetomidine versus standard therapy with fentanyl for sedation in mechanically ventilated premature neonates. J Pediatr Pharmacol Ther. 2012;17(3):252-62.

32. Malk K, Metsäranta M, Vanhatalo S. Drug effects on endogenous brain activity in preterm babies. Brain Dev. 2014;36(2):116-23.

33. Young GB, da Silva OP. Effects of morphine on the electroencephalograms of neonates: a prospective, observational study. Clin Neurophysiol. 2000; 111(11):1955-60.

34. Kubota T, Fukasawa T, Kitamura E, Magota M, Kato Y, Natsume J, et al. Epileptic seizures induced by dexmedetomidine in a neonate. Brain Dev. 2013;35(4):360-2.

35. van Alfen-van der Velden AA, Hopman JC, Klaessens JH, Feuth T, Sengers $\mathrm{RC}$, Liem KD. Effects of midazolam and morphine on cerebral oxygenation and hemodynamics in ventilated premature infants. Biol Neonate. 2006; 90(3):197-202

36. Young-McCaughan S, Miaskowski C. Definition of and mechanism for opioid-induced sedation. Pain Manag Nurs. 2001 Sep;2(3):84-97.

37. Kaur M, Singh PM. Current role of dexmedetomidine in clinical anesthesia and intensive care. Anes Essays Res. 2011;5(2):128-33.

38. Jamadarkhana S, Gopal S. Clonidine in adults as a sedative agent in the intensive care unit. J Anaesthesiol Clin Pharmacol. 2010;26(4):439-45.

39. Grunau RV, Craig KD. Pain expression in neonates: facial action and cry. Pain. 1987;28(3):395-410.

40. Lawrence J, Alcock D, McGrath P, Kay J, MacMurray SB, Dulberg C. The development of a tool to assess neonatal pain. Neonatal Netw. 1993;12(6): 59-66.

41. Stevens B, Johnston C, Petryshen P, Taddio A. Premature infant pain profile: development and initial validation. Clin J Pain. 1996;12(1):13-22.

42. Stevens BJ, Gibbins S, Yamada J, Dionne K, Lee G, Johnston C, et al. The premature infant pain profile-revised (PIPP-R): initial validation and feasibility. Clin J Pain. 2014;30(3):238-43.

43. Hummel P, Puchalski M, Creech SD, Weiss MG. Clinical reliability and validity of the N-PASS: neonatal pain, agitation and sedation scale with prolonged pain. J Perinatol. 2008;28(1):55-60.

44. Krechel SW, Bildner J. CRIES: a new neonatal postoperative pain measurement score. Initial testing of validity and reliability. Paediatr Anaesth. 1995:5(1):53-61.

45. Debillon T, Zupan V, Ravault N, Magny JF, Dehan M. Development and initial validation of the EDIN scale, a new tool for assessing prolonged pain in preterm infants. Arch Dis Child Fetal Neonatal Ed. 2001;85(1):F36-41.

46. Ista $E$, van Dijk M, Tibboel D, de Hoog M. Assessment of sedation levels in pediatric intensive care patients can be improved by using the COMFORT "behavior" scale. Pediatric critical care medicine: a journal of the Society of Critical Care Medicine and the World Federation of Pediatric Intensive and Critical Care Societies. 2005;6(1):58-63.

47. van Dijk M, Roofthooft DW, Anand KJ, Guldemond F, de Graaf J, Simons S, et al. Taking up the challenge of measuring prolonged pain in (premature) neonates: the COMFORTneo scale seems promising. Clin J Pain. 2009;25(7): 607-16.

48. Lundqvist P, Kleberg A, Edberg AK, Larsson BA, Hellstrom-Westas L, Norman E. Development and psychometric properties of the Swedish ALPS-Neo pain and stress assessment scale for newborn infants. Acta Paediatr. 2014; 103(8):833-9.

49. Review Manager 5. 5.3 ed. Copenhagen: Nordic Cochrane Centre: The Cochrane Collaboration; 2014.

50. Cochrane Handbook for Systematic Reviews of Interventions: Cochrane; 2019. Available from: www.training.cochrane.org/handbook.

51. Sterne JA, Hernán MA, Reeves BC, Savović J, Berkman ND, Viswanathan M et al. ROBINS-I: a tool for assessing risk of bias in non-randomised studies of interventions. BMJ (Clinical research ed). 2016;355:14919.

52. Higgins JP, Thompson SG, Deeks JJ, Altman DG. Measuring inconsistency in meta-analyses. BMJ (Clinical research ed). 2003:327(7414):557-60.

53. Egger M, Davey Smith G, Schneider M, Minder C. Bias in meta-analysis detected by a simple, graphical test. BMJ (Clinical research ed). 1997; 315(7109):629-34

54. Handbook for grading the quality of evidence and the strength of recommendations using the GRADE approach: GRADE working group; 2013 Available from: gdt.gradepro.org/app/handbook/handbook.html.

55. Regulation (EC) No 1901/2006 of the European Parliament and of the Council of 12 December 2006 on medicinal products for paediatric use and amending Regulation (EEC) No 1768/92, Directive 2001/20/EC, Directive 2001/83/EC and Regulation (EC) No 726/2004 (2019).

56. Pillai GC, Mentré F, Steimer JL. Non-linear mixed effects modeling - from methodology and software development to driving implementation in drug development science. J Pharmacokinet Pharmacodyn. 2005;32(2):16183.

\section{Publisher's Note}

Springer Nature remains neutral with regard to jurisdictional claims in published maps and institutional affiliations.
Ready to submit your research? Choose BMC and benefit from:

- fast, convenient online submission

- thorough peer review by experienced researchers in your field

- rapid publication on acceptance

- support for research data, including large and complex data types

- gold Open Access which fosters wider collaboration and increased citations

- maximum visibility for your research: over $100 \mathrm{M}$ website views per year

At BMC, research is always in progress.

Learn more biomedcentral.com/submissions 